\title{
Ultrasound-guided percutaneous carpal tunnel release in patients on hemodialysis: early experiences and clinical outcomes
}

This article was published in the following Dove Press journal:

Therapeutics and Clinical Risk Management

\author{
Ping-Hui Wang' \\ Chia-Lung $\mathrm{Li}^{2,3}$ \\ Chung-Jung Shao ${ }^{4}$ \\ Kuo-Chen $\mathrm{Wu}^{5}$ \\ Tai-Chang Chern ${ }^{6}$ \\ I-Ming Jou ${ }^{7,8}$
}

'Department of Orthopedics, Chi-Mei Medical Center, Tainan 710, Taiwan; ${ }^{2}$ Department of Physical Therapy, College of Medicine, National Cheng Kung University, Tainan, 70I, Taiwan;

${ }^{3}$ Department of Orthopedics, National Cheng Kung University Hospital, College of Medicine, National Cheng Kung University, Tainan 704, Taiwan;

${ }^{4}$ Department of Orthopedics, Tainan Municipal Hospital, Tainan 709, Taiwan; ${ }^{5}$ Department of Orthopedics, Kuo General Hospital, Tainan, Taiwan; ${ }^{6}$ Chern Tai-Chang Orthopedic Clinic, Pingtung 900, Taiwan; ${ }^{7}$ Department of Orthopedics, E-Da Hospital, I-Shou University, Kaohsiung, Taiwan; ${ }^{8}$ Department of Orthopedics, National Cheng Kung University, Tainan, Taiwan

Correspondence: I-Ming Jou Department of Orthopedics, E-Da Hospital, I-Shou University, I Yi-Da Road, Jiao-Su, Yen-Chao, Kaohsiung, Taiwan

Tel +8 $867615001 \mathrm{I}$

Fax +8 8676155581

Email ed109325@edah.org.tw
Purpose: The purpose of the current study was to evaluate the effectiveness of ultrasoundguided percutaneous carpal tunnel release in hemodialysis patients with carpal tunnel syndrome. Methods: From February 2009 to April 2013, a prospective review of 113 consecutive cases of ultrasound-guided percutaneous carpal tunnel release was carried out in 84 hemodialysis patients. Results were analyzed by clinical subjective scale, two self-administered questionnaires, and functional evaluations at seven time points (1 week and 1, 3, 6, 12, 18, and 24 months).

Results: Satisfactory symptom improvement in patients was $82 \%, 80 \%, 86 \%, 89 \%, 90 \%$, $91 \%$, and $90 \%$ at 1 week and $1,3,6,12,18$, and 24 months postoperatively, respectively. Moderate pain was suffered in $11.5 \%$ of patients within 1 week, $8.8 \%$ within 1 month, $2.7 \%$ within 3 months, and none after 12 months postoperatively. Static two-point discrimination and Semmes-Weinstein monofilament examinations presented significant improvements after 1 week and 1 month postoperatively and with time. Postoperative grip power demonstrated recovery and a significant increase after 3 and 6 months postoperatively. Three-jaw chuck-pinch strength showed significant increase after 1 month postoperatively. There were no operative complications.

Conclusion: Ultrasound-guided percutaneous carpal tunnel release is an effective and safe procedure in hemodialysis patients with carpal tunnel syndrome. The advantages include a less invasive procedure, no tourniquet needed, only limited infiltration anesthesia, minimal soft-tissue exploration, and relatively short operation time. Our data suggest this technique can reliably relieve clinical symptoms, with early restoration of grip and pinch strength.

Keywords: carpal tunnel, hemodialysis, ultrasound, percutaneous release

\section{Introduction}

Carpal tunnel syndrome (CTS) is a frequent complication of patients undergoing hemodialysis. It often causes pain, numbness, tingling, or burning sensation in the median nerve distribution, hand weakness, and reduced quality of life. ${ }^{1,2,3}$ CTS in hemodialysis patients is recognized as an unremitting course, and surgical release procedures are often required after a long period of hemodialysis. ${ }^{4,5}$

Traditionally, open carpal tunnel release (OCTR) is used in CTS patients on longterm hemodialysis, and has the advantage of direct and complete release of pathogens. $^{2}$ Friable skin, arteriovenous fistula (AVF), and coagulopathy make OCTR and extended OCTR prone to create more morbidities in hemodialysis patients than in the general population. ${ }^{6}$ Endoscopic CTR (ECTR) has been applied from idiopathic CTS to hemodialysis-related CTS. Although ECTR has superior short-term 
results, final effectiveness is the same between idiopathic and long-term hemodialysis patients at 3-6 months postoperatively. ${ }^{7}$ However, the potential risk of incomplete release and injury of adjacent neurovascular structures restrict application of ECTR in idiopathic and long-term hemodialysis patients. ${ }^{3,8}$

Ultrasound is a safe image modality and applied in many medical procedures. Advances in high-frequency musculoskeletal ultrasound have made related therapeutic applications in many musculoskeletal pathogens possible. ${ }^{9}$ The structure of the carpal tunnel, including the transverse carpal ligament (TCL), medial nerve, flexor tendons, proximal palmar arch, and ulnar artery, are easily visible using high-frequency ultrasound. Many studies have recently reported ultrasound application in CTR, from cadaver studies to clinical cases. ${ }^{10-14}$ Chern et al presented their clinical experiences and outcomes of ultrasound-guided percutaneous CTR (PCTR). This has proved to be a effective and safe procedure for idiopathic CTS. ${ }^{11}$ Nonetheless, there have been few studies of the application of ultrasound-guided PCTR in hemodialysis patients. The purpose of the current work is thus to present the results of ultrasound-guided PTCR in a consecutive series of hemodialysis patients with CTS.

\section{Methods}

\section{Patient population}

The study was reviewed and approved by the Ethics Committee of National Cheng Kung University Hospital. All participants were aware of the objective of this surgery and signed informed-consent documents. This study was conducted in accordance with the Declaration of Helsinki.

From February 2009 to April 2013, we performed 113 ultrasound-guided PCTRs in 84 hemodialysis patients with CTS. Initial data collected were age, sex, duration of disease, duration of hemodialysis, involved hand, symptom frequency, provocative factors, and comorbidity. CTS was diagnosed in accordance with clinical symptoms of pain, paresthesia and numbness in median nerve distribution, clumsiness, weakness of the hand, positive stimulation tests of Tinel's sign, flick sign, Phalen's test, and positive electrophysiological test results. The indication for surgery was any hemodialysis patient with CTS who had failed on conservative treatment $>3$ months or clinical evidence of thenar muscle atrophy or weakness. We excluded patients with acute CTS, recurrent CTS, inflammatory arthropathy, and documented peripheral neuropathy from the study.

\section{Operative procedures}

All patients had hemodialysis the day before surgery. They all received the same operative procedure: ultrasound guided PTCR under local anesthesia without use of a tourniquet. Firstly, flexor tendons, the TCL, median nerve, and bone landmarks were localized by an ultrasound scanner (5-10 MHz; SonoSite, Bothell, WA, USA) and safe zones determined. Secondly, insertion of the 21-gauge needle for $1 \%$ lidocaine infiltration and then creation of an inlet with an 18-gauge needle for the probe was done. Thirdly, the custom-made blunt probe was inserted subcutaneously to create the correct tract, and the hook knife (Ectra; Smith and Nephew, Andover, MA,USA) was introduced to cut the TCL backwardly. After cutting, the probe was inserted to evaluate adequate release. Real-time ultrasound was used to monitor all these procedures ${ }^{11}$ (Figure 1).

\section{Postoperative care}

The small puncture wound of the inlet was covered with a dry dressing without suturing postoperatively. The dressing was able to be removed in the next day. These patients were permitted to engage in all their daily activities and works as tolerated.

\section{Assessing results and outcomes}

Arrangements were made for patients to return to the hospital 1 week and $1,3,6,12,18$, and 24 months after the operation. Postoperative assessments were performed by an experienced orthopedic surgeon and a hand therapist. The assessment consisted of an interview with selfadministered questionnaires and a clinical examination, including the same strength and sensitivity measurements.

Postoperatively, subjective improvement in symptoms was rated from three grades (poor, fair,and good). Pre- and postoperatively, patients completed the Carpal Tunnel Syndrome Symptom Severity Score (CTS-SSS) and Carpal Tunnel Syndrome Functional Status Score (CTS-FSS) questionnaires, which scaled the patients' symptoms from 1 to 5 , with 5 representing the greatest symptoms or the most functional difficulty. ${ }^{15}$ Mean scores were recorded.

Digital sensibility was assessed by static two-point discrimination and Semmes-Weinstein monofilament tests over the proximal and distal phalanges of the thumb, middle and distal phalanges of the index, middle, and ring fingers, and the little finger for comparison. The grip strength of involved and uninvolved hands was assessed by all five settings of a hand dynamometer 
A
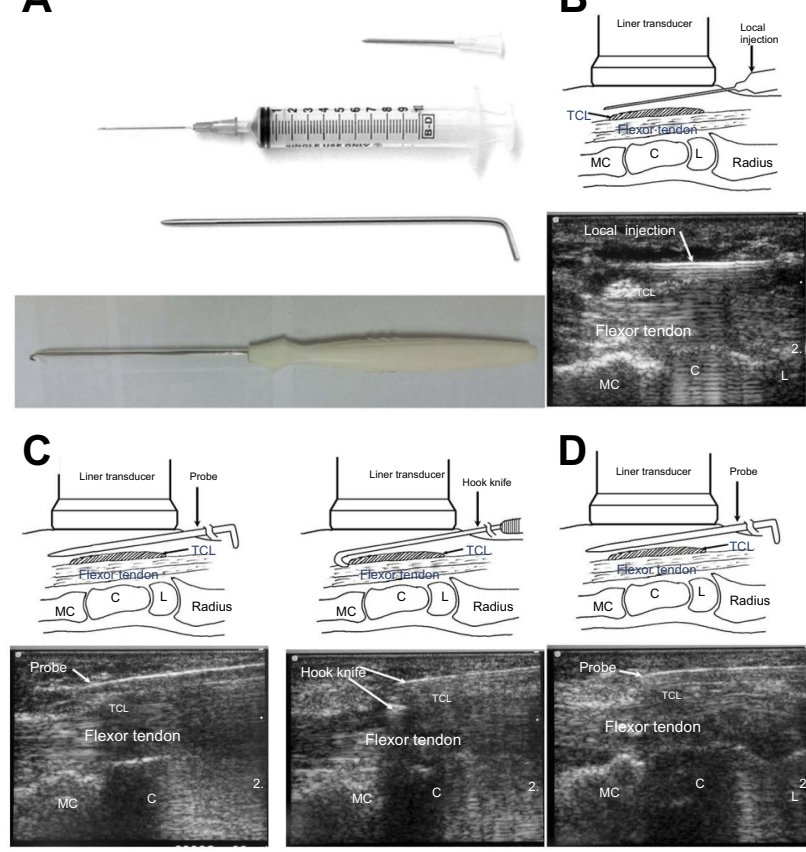

B

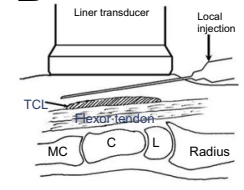

D

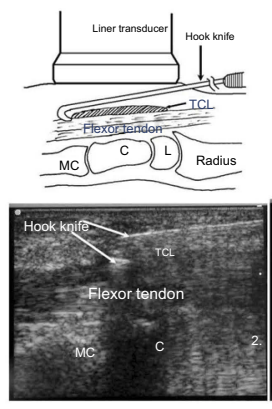

D

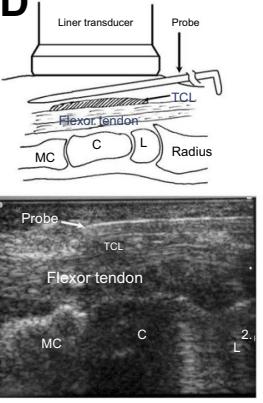

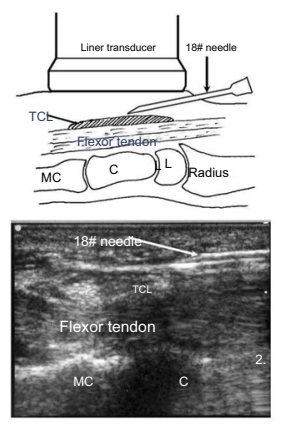
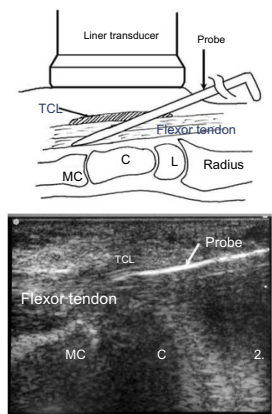

Figure I (A) Instruments for ultrasound-guided percutaneous carpal tunnel release: 18-gauge needle, $10 \mathrm{~mL}$ syringe with 21 -gauge needle, custom-made blunt probe, custom-made hook knife. (B) Insertion of the 21 -gauge needle for lidocaine infiltration and then to create an inlet with an I8-gauge needle. (C) The custom-made blunt probe was inserted subcutaneously to create the correct tract, and the hook knife was introduced to cut the TCL backwardly. (D) The probe was inserted to evaluate adequate release.

Abbreviations: C, capitates; L, lunate; MC, metacarpal; TCL, transverse carpal ligament.

(Asimov Engineering, LA, CA, USA). We used the maximum grip strength for statistical analysis. Three-jaw chuck-pinch strengths were measured using a pinch meter (Therapeutic Instruments, Clifton, NJ, USA).

\section{Statistical analysis}

All measurements are expressed as means $\pm \mathrm{SD}$. GraphPad (La Jolla, CA, USA) Prism 5.0 software was used to analyze the different graded areas. One-way ANOVA was used to analyze the measurements, and post hoc pair-wise comparisons were performed in cases of significant difference. Statistical significance was set at $P<0.05$.

\section{Results}

This prospective study consisted of 113 consecutive primary ultrasound-guided PCTR cases performed in 84 hemodialysis patients, including 56 women (67\%) and 28 men (33\%; age range $39-75$ years, average age $60.9 \pm 9.17$ years) during February 2009 to April 2013. The duration of patients on hemodialysis averaged $7.53 \pm 3.23(1.0-15)$ years. The duration of symptoms averaged $24.3 \pm 29.8(0.5-120)$ months. Diagnosis was recorded as bilateral CTS in 34 patients (40\%, 21 women and 13 men). CTS involved the AVF hand in $75(89 \%)$ patients. Before surgery, 42 (37\%) hands had thenar muscle atrophy, 28 (25\%) hands suffered from symptoms all day, and 64 (57\%) frequently. A total of 64 (57\%) hands had symptoms that intensified during sleep, and $49(43 \%)$ with repetitive movements of the wrist. In sum, 49 (58\%) hands had positive Phalen's tests, 63 (75\%) positive Tinel's sign, and eight (10\%) neither of these. Comorbidities included 61 (73\%) patients with trigger finger, $56(66 \%)$ with hypertension, $34(41 \%)$ with diabetes, and $12(14 \%)$ with hyperlipidemia. The 34 recruited diabetic patients had no concomitant evidence of peripheral neuropathy. All patients tolerated the procedure, and division of the TCL was confirmed by ultrasound postoperatively. Complete preoperative data were accessible for all cases. All patients underwent complete follow-up by reexamination or telephone.

\section{Symptom relief, postoperative pain, and tenderness}

Subjective sensory disturbances, such as paresthesia and hypoesthesia, improved immediately postoperatively, and such sensory disturbances had dissolved in $82 \%, 80 \%, 86 \%$, $89 \%, 90 \%, 91 \%$, and $90 \%$ of cases at 1 week and $1,3,6,12$, 18 , and 24 months after the operation, respectively. Evaluation of pain at each follow-up is described in Table 1. Average CTS-SSS was 3.49 and CTS-FSS 3.73 preoperatively. Both 
Table I Survey scale of symptom improvement after ultrasound-guided percutaneous release

\begin{tabular}{|l|l|l|l|l|l|l|l|}
\hline & I week & I month & $\mathbf{3}$ months & $\mathbf{6}$ months & I2 months & I 8 months & $\mathbf{2 4}$ months \\
\hline Poor & $7 \%$ & $8 \%$ & $5 \%$ & $3 \%$ & $1 \%$ & $1 \%$ & $1 \%$ \\
Fair & $11 \%$ & $12 \%$ & $9 \%$ & $8 \%$ & $9 \%$ & $8 \%$ & $9 \%$ \\
Good & $82 \%$ & $80 \%$ & $86 \%$ & $89 \%$ & $90 \%$ & $91 \%$ & $90 \%$ \\
\hline
\end{tabular}

scores improved continuously throughout the follow-up period. Moderate numbness occurred in $32.7 \%$ of patients within 1 week, $21.2 \%$ within 1 month, $7 \%$ within 3 months, and no patients 18 months after the operation. Moderate pain occurred in $11.5 \%$ of patients within 1 week, $8.8 \%$ within 1 month, $2.7 \%$ within three months, and no patients 12 months after the operation. Ultimately, average CTS-SSS improved to 1.18 and CTS-FSS to 1.00 at 24 months postoperatively (Table 2).

\section{Sensory testing and strength}

A total of 91 ( 91 of $113,70.5 \%$ ) hands revealed $>6 \mathrm{~mm}$ in the two-point discrimination evaluation preoperatively. Postoperative scores (two-point discrimination $>6 \mathrm{~mm}$ ) decreased to $46.7 \%$ at 1 week and $4.9 \%$ at 24 months. Scores in both static two-point discrimination and Semmes-Weinstein monofilament examinations demonstrated significant improvements after 1 week and 1 month postoperatively and with time. Grip and threejaw chuck-pinch strength scores averaged $15.17 \pm 14.3$ and $4.87 \pm 2.1 \mathrm{~kg}$ preoperatively. Grip power had diminished at 1 week after operation, and then recovered and had significantly increased at 3- and 6-month follow-up examinations. Three-jaw chuck-pinch strength showed a significant increase after 1 month postoperatively. Grip and three-jaw chuck-pinch strength scores had increased to $17.11 \pm 10.6$ and $9.98 \pm 3.6 \mathrm{~kg}$ at the final follow-up. All these data imply the effect of the operations (Table 3 ).

\section{Wound healing and complications}

In all patients, the wound healed uneventfully within a few days and became invisible without other cosmetic problems. No patients had neurovascular structure/tendon injury or superficial/deep tendon-sheath infection.

\section{Final outcomes}

All 113 hands in this prospective study were assessed by clinical examination or telephone review based on the CTS-SSS and CTS-FSS. The satisfactory level of good symptom improvement of the patients was $90 \%$ at the final 24-month follow-up. No patient suffered from moderate hand or wrist pain after 1 year postoperatively according to the CTS-SSS. Recovery of and improvement in grip power was after 3 and 6 months postoperatively, respectively. Three-jaw chuck pinch had increased significantly after 1 month postoperatively.

\section{Discussion}

CTS in long-term hemodialysis patients has incidence varying from $2 \%$ to $32 \%$, which is higher than in the general population. When it occurs in renal dialysis, it is also recognized as more serious and unremitting. ${ }^{4,5}$ The etiology of CTS in long-term hemodialysis patients is unclear, and several factors relate to it, including duration of dialysis, age, old age of patient at onset of dialysis, dialysis-membrane characteristics, advanced glycosylation end products, low serum albumin, diabetic nephropathy, $\beta_{2}$ -microglobulin $\left(\beta_{2} \mathrm{MG}\right)$ clearance, and sex. ${ }^{5,16-18}$ In our study, female hemodialysis patients receiving surgery comprised $67 \%$, which is compatible with a Japanese cohort study, ${ }^{18}$ although other studies had different results, with an equal ratio or more of the male population. ${ }^{5,19}$ Dialysis-related $\beta_{2} \mathrm{MG}$ amyloidosis deposits are considered to connect with synovial tissue hyperplasia, TCL thickening and flexor tenosynovitis, and all these may cause CTS. There were $61(73 \%)$ patients that also had trigger finger in our study, and this may have been a coeffect of dialysis-related $\beta_{2} \mathrm{MG}$ amyloidosis. Some studies have emphasized that AVF has a local effect on the carpal tunnel in CTS, ${ }^{20,21}$ while AVF on arms with CTS show a higher blood-flow rate and double entrapment of the median nerve is associated with polytetrafluoroethylene loop graft. ${ }^{16}$ Other studies found no clear relationship between AVF and CTS. ${ }^{2,4,22}$ In the current work, $89 \%$ of the hemodialysis patients had CTS at the AVF site. Many systemic diseases contribute to and exacerbate the development of CTS, including rheumatoid arthritis, diabetes mellitus, gout, acromegaly, hypothyroidism, and amyloidosis. ${ }^{2,23}$ In this study, $41 \%$ patients had diabetes.

CTS caused by long-term hemodialysis has been recognized as a relatively advanced and incurable disease with acute progression. The rather limited postoperative improvement after CTR and the high recurrence rate would be expected in 

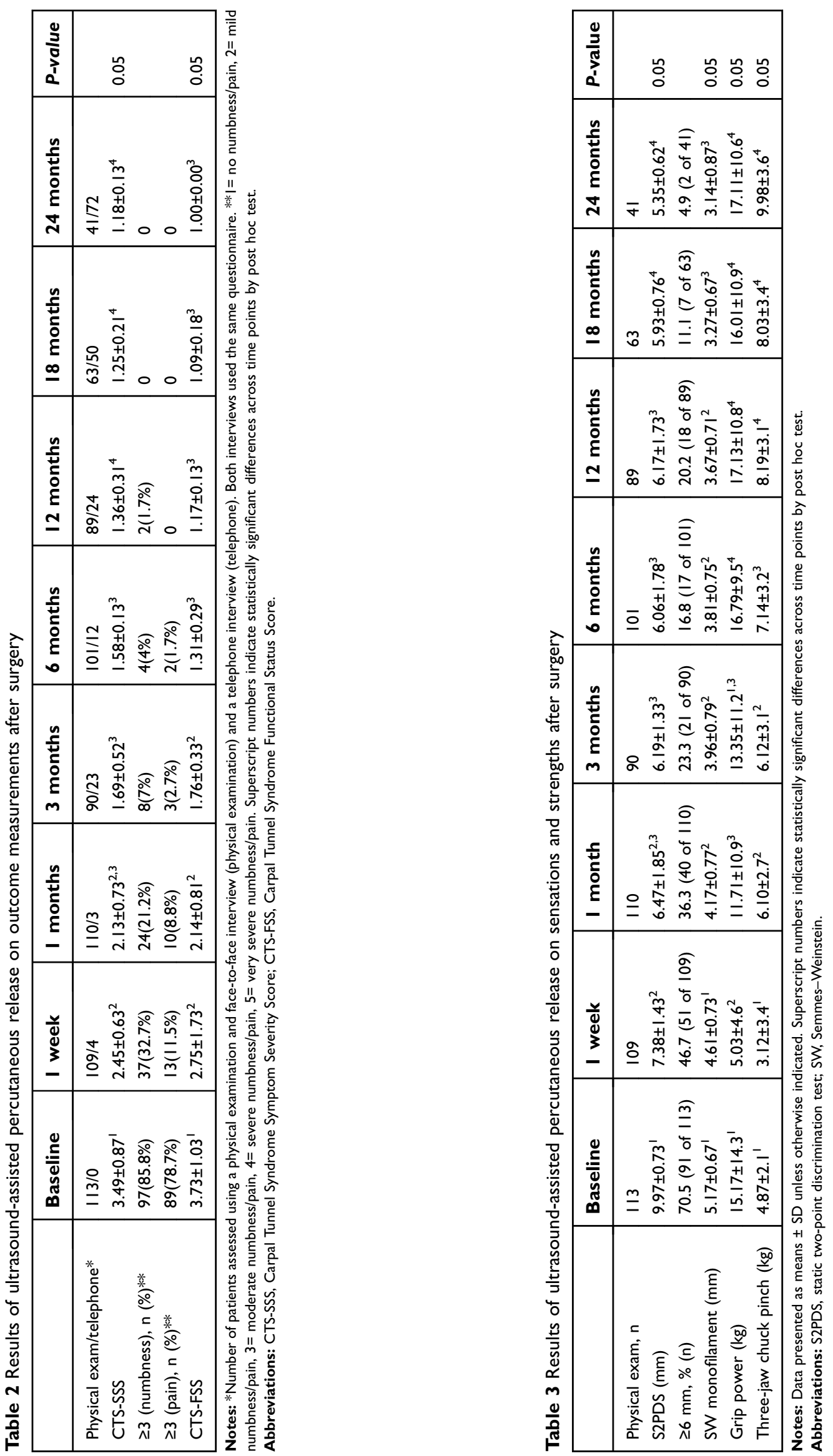
these patients. ${ }^{4,16,17}$ Surgical decompression of the carpal tunnel is suggested if conservative treatment has failed. ECTR, OCTR, and extended OCTR are usually used in these patients. Nonetheless, there are still no randomized trials or wellestablished guidelines for treating CTS in long-term hemodialysis patients. Theoretically extended or standard OCTR may enable better view and complete decompression of the median nerve. Neurolysis and synovectomy can be done in the same procedure, although the effects remain controversial. ${ }^{7,24}$ ECTR is another alternative, with advantages of less postoperative pain, faster return to work, and fewer woundrelated complications. One study revealed that ECTR was effective for idiopathic and long-term hemodialysis patients, but with less satisfactory electrophysiological outcomes in the latter. $^{7}$ A meta-analysis compared OCTR and ECTR and found that the final results were the same. ${ }^{8}$ However, the restricted endoscopic views of the anatomic structures, incomplete decompression, and potential risk of nerve or vessel injury limit the use of ECTR. ${ }^{25-28}$

Ultrasound-assisted percutaneous release of trigger fingers is now a safe and easy procedure, ${ }^{29-31}$ and ultrasoundguided PCTR has been described in several studies with satisfactory preliminary results without injury to the surrounding structures. ${ }^{11,14,32-34}$ In a pilot open-label uncontrolled trial of 39 patients with ultrasound-guided PCTR, Locoq et al observed scores for pain, formication, and discomfort experienced in the hand had significantly reduced by day $15 .^{32}$ Chen et al presented results of ultrasoundguided PCTR in 91 CTS cases with $100 \%$ sensory disturbances, and showed $98.9 \%$ moderate pain disappearance in 12 months postoperatively without intra- or postoperative complication. ${ }^{11}$ Another study involving $128 \mathrm{CTS}$ patients who received ultrasound-guided PCTR reported significant improvement in symptom severity and functional status scores 6 months postoperatively. Follow-up magnetic resonance imaging showed a complete section of TCL and nerve decompression in all patients. ${ }^{33}$ In a randomized clinical trial to compare ultraminimally ultrasound-guided PCTR and blind mini-OCTR, Rojo-Manaute et al found the former provided early functional return and less postoperative morbidity with the same neurological recovery. ${ }^{34}$ The main advantages of ultrasound-guided PCTR are minimizing surgical damage to nonetiologic anatomic structures and accurately restricting release to the anatomic fibrous layer by small skin incisions, strong resolution, and real-time ultrasound monitoring. The tools of the technique are simple and inexpensive. The procedure has been proved to be safe and effective in cadaveric and clinical studies. ${ }^{10,11,32-34}$ The limitation of the procedure is that it is technically demanding. Nonetheless, the technical difficulty may be overcome with high-frequency ultrasound, advance cadaver practice, and repeated preoperative skills training.

To our knowledge, there have been no previous clinical reports for ultrasound-guided PCTR in hemodialysis patients. In this study, $90 \%$ of the patients had subjectively good results with improved symptoms. All objective parameters (CTS-SSS, CTS-FSS, grip power, three-jaw chuckpinch strength, Semmes-Weinstein monofilament tests, and two-point discrimination tests) showed progressive and significant improvements during 2 years of follow-up. The difference between subjective and objective satisfaction may be due to preoperative expectations of patients for surgery.

There are some limitations regarding this study. There was no comparative group to compare different surgical strategies. Also, there was no collection of complete follow-up data for the clinical examination $(78.7 \%$ and $36.3 \%$ at 12 and 24 months, respectively), although there were telephone interviews using questionnaires (100\%).

\section{Conclusion}

Ultrasound-guided PCTR is an effective and safe procedure in hemodialysis patients with CTS. Advantages include a less invasive procedure, no tourniquet needed, limited-infiltration anesthesia, minimal soft-tissue exploration, and relatively short operation time. Our data suggested this technique can reliably relieve clinical symptoms and restore grip and pinch strength. Ultrasoundguided PTCR is a safe and effective procedure in hemodialysis patients with CTS.

\section{Disclosure}

The authors report no conflicts of interest in this work.

\section{References}

1. Schwarz A, Keller F, Seyfert S, Pöll W, Molzahn M, Distler A. Carpal tunnel syndrome: a major complication in long-term hemodialysis patients. Clin Nephrol. 1984;22(3):133-137.

2. Al-Benna S, Nano PG, El-Enin H. Extended open-carpal tunnel release in renal dialysis patients. Saudi J Kidney Dis Transpl. 2012;23 (6):1181-1187. doi:10.4103/1319-2442.103557

3. Kang HJ, Koh IH, Lee WY, Choi YR, Hahn SB. Does carpal tunnel release provide long-term relief in patients with hemodialysis-associated carpal tunnel syndrome? Clin Orthop Relat Res. 2012;470(9):2561-2565. doi:10.1007/s11999-012-2309-4

4. Wilson SW, Pollard RE, Lees VC. Management of carpal tunnel syndrome in renal dialysis patients using an extended carpal tunnel release procedure. J Plast Reconstr Aesthet Surg. 2008;61 (9):1090-1094. doi:10.1016/j.bjps.2007.07.004 
5. Kopeć J, Gadek A, Drozdz M, et al. Carpal tunnel syndrome in hemodialysis patients as a dialysis-related amyloidosis manifestation-incidence, risk factors and results of surgical treatment. Med Sci Monit. 2011;17(9):CR505-CR5059. 10.12659/MSM.881937

6. Kim SJ, Shin SJ, Kang ES. Endoscopic carpal tunnel release in patients receiving long-term hemodialysis. Clin Orthop Relat Res. 2000;376:141-148. doi:10.1097/00003086-200007000-00020

7. Ho SYC, Lin YT. Comparing the effectiveness of endoscopic carpal tunnel release between idiopathic and long-term hemodialysis patients. Formosan J of Surg. 2014;47(1):11-17. doi:10.1016/j.fjs.2013.05.007

8. Sayegh ET, Strauch RJ. Open versus endoscopic carpal tunnel release: a meta-analysis of randomized controlled trials. Clin Orthop Relat Res. 2015;473(3):1120-1132. doi:10.1007/s11999-014-3835-Z

9. Colio SW, Smith J, Pourcho AM. Ultrasound-guided interventional procedures of the wrist and hand: anatomy, indications, and techniques. Phys Med Rehabil Clin N Am. 2016;27(3):589-605. doi:10.1016/j.pmr.2016.04.003

10. Chern TC, Jou IM, Chen WC, Wu K-C, Shao C-J, Shen P-C. An ultrasonographic and anatomical study of carpal tunnel, with special emphasis on the safe zones in percutaneous release. J Hand Surg Eur Vol. 2009;34(1):66-71. doi:10.1177/1753193408097322

11. Chern TC, Kuo LC, Shao CJ, Wu TT, Wu K-C, Jou I-M. Ultrasonographically guided percutaneous carpal tunnel release: early clinical experiences and outcomes. Arthroscopy. 2015;31 (12):2400-2410. doi:10.1016/j.arthro.2015.06.023

12. Markison RE. Percutaneous ultrasound-guided MANOS carpal tunnel release technique. Hand. 2013;8(4):445-449. doi:10.1007/s11552-0139554-1

13. de la Fuente J, Miguel-Perez MI, Balius R, Guerrero V, Michaud J, Bong D. Minimally invasive ultrasound-guided carpal tunnel release: a cadaver study. J Clin Ultrasound. 2013;41(2):101-107. doi:10.1002/ jcu. 21982

14. Ohno K, Hirofuji S, Fujino K, Ishidu T, Kira S, Neo M. Sonographic monitoring of endoscopic carpal tunnel release. J Clin Ultrasound. 2016;44(9):597-599. doi:10.1002/jcu.22398

15. Levine DW, Simmons BP, Koris MJ, et al. A self-administered questionnaire for the assessment of severity of symptoms and functional status in carpal tunnel syndrome. J Bone Joint Surg Am. 1993;75:1585-1592. doi:10.2106/00004623-199311000-00002

16. Charra B, Calemard E, Laurent G. Chronic renal failure treatment duration and mode: their relevance to the late dialysis periarticular syndrome. Blood Purif. 1988;6(2):117-124. doi:10.1159/000169493

17. Jadoul M. Dialysis-related amyloidosis: importance of biocompatibility and age. Nephrol Dial Transplant. 1998;13(Suppl 7):61-64.

18. Hoshino J, Yamagata K, Nishi S, et al. Carpal tunnel surgery as proxy for dialysis-related amyloidosis: results from the Japanese society for dialysis therapy. Am J Nephrol. 2014;39(5):449-458. doi:10.1159/000362567

19. Benz RL, Siegfried JW, Teehan BP. Carpal tunnel syndrome in dialysis patients: comparison between continuous ambulatory peritoneal dialysis and hemodialysis populations. Am J Kidney Dis. 1988;11(6):473-476. 10.1016/S0272-6386(88)80082-9
20. Gousheh J, Iranpour A. Association between carpel tunnel syndrome and arteriovenous fistula in hemodialysis patients. Plast Reconstr Surg. 2005;116(2):508-513. 10.1097/01.prs.0000172893.59458.8a

21. Gargouri-Berrechid A, Sidhom Y, Lanouar L, et al. The arteriovenous fistula is an additional risk factor for developing carpal tunnel syndrome in hemodialysis patients. Nephrol Ther. 2014;10(3):177-180. doi:10.1016/j.nephro.2014.01.008

22. Jain VK, Cestero RV, Baum J. Carpal tunnel syndrome in patients undergoing maintenance hemodialysis. Jama. 1979;242(26):2868-2869. 10.1001/jama.1979.03300260038024

23. Lin HH, Chen HT, Chen YC, et al. Carpal tunnel syndrome in long-term hemodialysis patients. Acta Nephrologica. 2001;15:111-114.

24. Shiota E, Tsuchiya K, Yamaoka K, Kawano O. Open carpal tunnel decompression in long-term haemodialysis patients. J Hand Surg Br. 2001;26(6):529-532. doi:10.1054/jhsb.2000.0550

25. Thoma A, Veltri K, Haines T, Duku E. A meta-analysis of randomized controlled trials comparing endoscopic and open carpal tunnel decompression. Plast Reconstr Surg. 2004;114(5):1137-1146. 10.1097/01.PRS.0000135850.37523.D0

26. Benson LS, Bare AA, Nagle DJ, Harder VS, Williams CS, Visotsky JL. Complications of endoscopic and open carpal tunnel release. Arthroscopy. 2006;22(9):919-924. doi:10.1016/j.arthro.2006.05.008

27. Vasiliadis HS, Xenakis TA, Mitsionis G, Paschos N, Georgoulis A. Endoscopic versus open carpal tunnel release. Arthroscopy. 2010;26 (1):26-33. doi:10.1016/j.arthro.2009.06.027

28. Nagle DJ, Fischer TJ, Harris GD, et al. A multicenter prospective review of 640 endoscopic carpal tunnel releases using the transbursal and extrabursal chow techniques. Arthroscopy. 1996;12(2):139-143. 10.1016/S0749-8063(96)90001-8

29. Jou IM, Chern TC. Sonographically assisted percutaneous release of the a1 pulley: a new surgical technique for treating trigger digit. J Hand Surg Br. 2006;31(2):191-199. doi:10.1016/j.jhsb.2005.11.003

30. Wu KC, Chern TC, Jou IM. Ultrasound-assisted percutaneous trigger finger release: it is safe. Hand. 2009;4(3):339. doi:10.1007/s11552009-9179-6

31. Sato ES, Dos Santos JB, Belloti JC, Albertoni WM, Faloppa F. Percutaneous release of trigger fingers. Hand Clin. 2014;30 (1):39-45. doi:10.1016/j.hcl.2013.08.017

32. Lecoq B, Hanouz N, Morello R, et al. Ultrasound-assisted surgical release of carpal tunnel syndrome: results of a pilot open-label uncontrolled trial conducted outside the operating theatre. Joint Bone Spine. 2015;82(6):442-445. doi:10.1016/j.jbspin.2015.01.024

33. Petrover D, Silvera J, De Baere T, Vigan M, Hakimé A. Percutaneous ultrasound-guided carpal tunnel release: study upon clinical efficacy and safety. Cardiovasc Intervent Radiol. 2017;40(4):568-575. doi:10.1007/s00270-016-1545-5

34. Rodriguez-Maruri G, Capa-Grasa A, Sanz-Ruiz P, et al. Ultra-minimally invasive ultrasound-guided carpal tunnel release: a randomized clinical trial. J Ultrasound Med. 2016;35(6):1149-1157. doi:10.7863/ ultra.15.07001
Therapeutics and Clinical Risk Management

\section{Publish your work in this journal}

Therapeutics and Clinical Risk Management is an international, peerreviewed journal of clinical therapeutics and risk management, focusing on concise rapid reporting of clinical studies in all therapeutic areas outcomes, safety, and programs for the effective, safe, and sustained use of medicines. This journal is indexed on PubMed Central, CAS,
Dovepress

EMBase, Scopus and the Elsevier Bibliographic databases. The manuscript management system is completely online and includes a very quick and fair peer-review system, which is all easy to use. Visit http://www.dovepress.com/testimonials.php to read real quotes from published authors. 